\title{
Neuroendocrine Tumuors of the Pancreas - Surgical Treatment and Prognostic Factors
}

\author{
Tsonka Lukanova, Cvetan Trichkov, Vasil Mihaylov, Ivelin Takorov, Maria Yakova, \\ Radoslav Kostadinov, Nikola Vladov
}

Department of HPB and Transplantation Surgery, Military Medical Academy, Sofia, Bulgaria

\section{ABSTRACT}

Background: Surgery is still the only curative treatment for patients with pancreatic neuroendocrine tumours (pNETs). Prognosis depends mainly on tumour characteristics.

Methods: The single-centred retrospective cohort study consisted of 58 patients with a histological confirmation of "pNET", who were operated over a time span of 10 years. The analysis is focused on the type of surgery, post-operative morbidity, survival rates and prognostic factors.

Results: Median age at diagnosis was 53.28 years (SD 9.85; 21-81). The operative interventions performed were radical in $79.31 \%$ of the patients and palliative in $20.69 \%$ of them, respectively. The overall survival rate was 63.41 months for the non-functioning pNETs versus 128.21 months for the functioning pNETs (Log Rank test, $p=0.036$ ). Upon applying Cox-regression, the only factors that were found as statistically significant predictors of poor outcome were non-functional status, distant metastases, vascular resection and lymphovascular invasion.

Conclusions: Radical surgery must be considered in all cases when it is oncologically consistent and technically achievable. Non-functional status, distant metastases, vascular resection and lymphovascular invasion are found to be associated with decreased survival. Key words: pancreatic neuroendocrine tumours, surgery, prognostic factors

\section{INTRODUCTION}

Pancreatic neuroendocrine tumors (pNETs) are rare neoplasms, comprising approximately $2 \%$ of all tumours of the pancreas, with a gradually increasing incidence during the last several decades (1). Based on the hormonal overproduction causing clinical syndromes, pNETs are divided in functional- (F-pNETs) and non-functional pNETs (NF-pNETs), insulinomas and gastrinomas being the most common F-pNETs (2). Efficient therapy requires a multidisciplinary healthcare team, while the prognosis is still ambiguous, depending mainly on the tumour characteristics. At present, surgery is still a primary treatment option. However, certain debatable aspects regarding the types of operative procedures and prognostic factors
Corresponding author: Tsonka Lukanova, MD Clinic of hepato-biliary-pancreatic Surgery and Transplantology Military Medical Academy, Sofia, 1606 3, Georgi Sofiiski, str., Bulgaria E-mail: tsonkalukanova@gmail.com
Received: 05.01.2019 Accepted: 04.02.2019 
associated with the tumour and its surgical treatment have recently arisen.

\section{MATERIAL AND METHODS}

In this retrospective cohort study, medical records of the patients who were operated in our centre between January 2008 and December 2017 with a histological diagnosis of "neuroendocrine pancreatic tumor" were reviewed. The collected data included demographic and tumour characteristics, type of surgery, postoperative morbidity, mortality and survival rates. The 2010 WHO classification (3) and the European Neuroendocrine Tumor Society - ENETS/TNM staging and grading systems $(4,5)$ were used to describe tumour characteristics.

The cohort was dichotomised in two groups of patients, depending on whether the tumour presented hormonal overproduction and its consequent clinical syndromes (F-pNETS) or not (NF-pNETs). Diagnoses were based on clinical presentation, preoperative imaging modalities (ultrasound - US, computed tomography scan - CT-scan, magnetic resonance imaging - MRI) and postoperative pathological formalin-fixed, paraffin-embedded tissue sections and immunohistochemical staining. All radical procedures except for tumour enucleation included standard regional lymph-node dissection (LND). Both conventional and laparoscopic procedures were performed.

Postoperative pancreatic fistula (POPF) was reported if clinically relevant - grade $B$ and grade $C$ according to the 2016 International Study Group definition and grading of postoperative pancreatic fistula (6). Postoperative bleeding was defined as early or late depending on whether it occurred in the first 24 hours postoperatively or later. Standard definitions were accepted for the other types of complications. Postoperative mortality was defined as death occurring within the first 30 post-operative days.

Data are presented as mean \pm standard error. Differences in the continuous variables of operative data were analyzed using variance and t-tests. Survival estimates were generated using the Kaplan-Meier method and the Log Rank test. Multivariate analysis by COX regression was used to examine the effects of prognostic factors. Differences with $p<0.05$ were considered to be statistically significant.

\section{RESULTS}

Between January 2008 and December 2017 fiftyeight patients with pathologically confirmed pancreatic neuroendocrine tumours underwent surgery in our Department. The demographic and tumour characteristics (size, staging and grading) are summarized in table 1 . There were 44 patients with NF-pNETs (75.86\%) and 14 patients with F-pNETs (24.14\%), with a mean age of 53.28 years (SD 9.85; 21-81). All tumours were solitary: 27 of them were located in the pancreatic head, 16 - in the pancreatic body and 17-in the pancreatic tail. All F-pNETs were benign (13 patients with insulinoma and only one gastrinoma). Specific hypersecretion outlined the clinical course in all patients with F-pNETs. Non-functional p-NETs manifested with abdominal pain and distension in $47.73 \%$ of the cases, abdominal mass $(20.45 \%)$, jaundice (27.27\%). 91.67\% had previously had a pre-operative biliary stent inserted or were diagnosed as incidentalomas in $18.18 \%$ of cases. The mean tumour size ofthe NF-pNETs was $5.17 \mathrm{~cm}$ (SD 3.19; 1.6-15), while for the F-pNET group it was $2.53 \mathrm{~cm}$ (SD 2.05:1.5-3.5) $(p=0.045)$.

All patients were preoperatively evaluated with abdominal ultrasound and high-resolution contrast CT scans. $57.14 \%$ of F-pNETs had secretin-stimulated MRI in order to measure tumour-to-main pancreatic duct distance and estimate the optimal type of resection to be employed. Only $31 \%$ of patients had preoperative biopsy. Intraoperative US was used in tumour localization in $15.38 \%$ of the insulinoma cases. Ki67\% was evaluated in all radically treated patients and G-status was defined. Neuron-specific enolase (NSE) was positive in $39.65 \%$, chromogranin - A (CgA) - in $67.24 \%$ and synaptophysin $63.79 \%$ of the cases, respectively. The "triple positive" combination (NSE, CgA and synaptophysin) was found in 19 out of 58 of the patients.

Table1 - Demographic and main tumor characteristics of NF-pNETs and F-pNETs groups

\begin{tabular}{|c|c|c|}
\hline Characteristic & NF-pNETs & F-pNETs \\
\hline $\mathrm{N}$, number & 44 & 14 \\
\hline Male & 19 & 4 \\
\hline Female & 25 & 10 \\
\hline Mean age, years & 57.86 (39-81; SD 9,83) & 48.71 (21-76; SD 9,88) \\
\hline Mean size, $\mathrm{cm}$ & 5.17 (1.6-15; SD 3.19) & 2.53 (1.5-8; SD 2.05) \\
\hline Stage IA+IB & 10 & 14 \\
\hline Stage IIA+IIB & 13 & - \\
\hline Stage III & 6 & - \\
\hline Stage IV & 15 & - \\
\hline NET G1 & 12 & 10 \\
\hline NET G2 & 12 & 4 \\
\hline NET G3 & 18 & 0 \\
\hline MANEC & 2 & 0 \\
\hline
\end{tabular}


The 58 patients underwent 60 operations. Two of them had liver metastases which were operated twice. One patient with synchronous liver metastases underwent laparoscopic distal spleno-pancreatectomy (DSP) at first stage for a pT3NOM1V1G2 - Ki 67 - 10\% pancreatic tail NET. Six months later after the somatostatin analogue treatment was concluded the patient had open left lobectomy with two liver metastasectomies (S6 and S8). 24 months after the intervention a follow-up SPECT-CT 99mTc-Tektrotyd scan revealed 2 small-sized S6 and S8 lesions. He died on the 38 months after the initial pancreatic resection. The other patient had a pylorus-preserving pancreato-duodenectomy (PPPD) for a PT2NOMOVO G1 - Ki 67 - $2 \%$ pancreatic head NET and 5 years later - a metastasectomy for a solitary metachronous liver metastasis. Six years postoperatively, he has multiple small-sized liver metastases which mandate Gallium-68 PET/CT and Peptide Receptor Radionuclide Therapy. Unfortunately these are currently unavailable in our country.

The operative procedures performed were RO-radical in 79.31\% of the cases and consisted of 19 DSP, 13 tumour enucleations, 11 pancreato-duodenectomies, 2 spleen-preserving distal pancreatectomies and 2 multivisceral resections. The remainder of $20.69 \%$ of the procedures were palliative ( 7 exploratory laparotomies and 5 palliative by-pass procedures) (fig. 1). Laparoscopic interventions were carried out in $25.86 \%$ of the cases and included 10 DSP, 4 enucleations and 1 exploratory laparotomy. While DSP was the most frequent procedure employed for the NF-pNET-group, standard LND and tumor enucleation was the main procedure done for the F-pNETs.
RO-liver resections were performed in $13.79 \%$ of the patients with synchronous $(n=7)$ metachronous $(n=1)$ liver metastases. $12.06 \%$ of the patients only had liver biopsy. There were $75 \%$ combined resections $-n=3$ - metastasectomies ( $n=1$ - solitary and $n=2$ - multiple) with DSP, $n=1$ - left lobectomy with metastasectomy and DSP, $\mathrm{n}=1$ - metastasectomy with a PPPD. The other $25 \%$ included one two-staged resection (DSP at first stage and left lobectomy with metastasectomies at second stage) and one solitary metastasectomy for a metachronous lesion on the 70-th month after a PPPD for a G1 NF-p NET. No specific liver resection complications were recorded. Vascular resections were performed in $5.17 \%$ of cases. These procedures included tangential resection of the portal vein and partial resection of the superior mesenteric vein, with end-to-side anastomosis in locally advanced NF-pNETs.

The overall postoperative morbidity rate was $46.5 \%$. The most common type of complication was clinicallyrelevant POPF, which occurred in 19 patients (32.7\%). There was also one biliary fistula, which was treated conservatively. Early postoperative bleeding occurred in 2 cases $(5.6 \%)$ while in one other patient the bleeding was late. All of these patients required relaparotomy. The other types of recorded complications were: pleural effusion (10.34\%), pancreatitis, (8.62\%), DGE (8.62\%), intraabdominal abscess $(8.62 \%)$ and surgical site infection (3.4\%). The postoperative mortality rate for the whole pNET-group was $5.2 \%$. All three deaths occurred in the NF-pNETs-patients' group, due to POPF type $\mathrm{C}$ and late bleeding.

The follow-up time ranged from 6 to 150 months, with a mean of $48.93 \pm 32.62$ months. The overall

Tumor enucleation

Spleen-preserving distal pancreatectomy

Distal splenopancreatectomy

Pancreato-duodenectomy

Pylorus-preserving-pancreatoduodenectomy

Multivisceral resection

Paliative by-pass

Exploration

$\begin{array}{llllllllll}0 & 2 & 4 & 6 & 8 & 10 & 12 & 14 & 16 & 18\end{array}$

F-NETs a NF-NETs

Figure 1 - Types of operative procedures in patients with NF-pNETs and F-pNETs 
survival rate was 63.41 months for the NF-pNETs vs 128.21 months for the F-pNETs (Log Rank test, $\mathrm{p}=0.036$ ) (fig. 2a). There was no significant difference in the survival rate of patients with pNETs $\leq 2 \mathrm{~cm}$, compared to pNETs > $2 \mathrm{~cm}(65.00 ; 95 \% \mathrm{Cl}, 28.79-101.21$ vs 61.15 ; $95 \% \mathrm{Cl}, 44.70-77.59)$ ( $p=0.753$ ) (fig. $2 b$ ), NO vs N1 (76.61 months vs 62.02 months; Log Rank test, $p=0.214)$, nor in the survival rate of patients with "triple positive - NSE, CgA, synaptophysin" compared to those that did not simultaneously express the three markers (102.01; $95 \% \mathrm{Cl}, 63.31-114.48$ vs $88.90 ; 95 \% \mathrm{Cl}, 44.70$ 77.59) ( $p=0.083$ ) (fig. $2 c)$. There was significant difference in the survival rate of patients with $\mathrm{G}$ or $\mathrm{G} 2$ pNETs, compared to $\mathrm{G} 3$ pNETs $(78.81 ; 95 \% \mathrm{Cl}$, 64.5793.06 vs $48.07 ; 95 \% \mathrm{Cl}, 23.19-72.94)$ ( $p=0.004$ ) (fig. $2 d$ ), as well as in the mean survival rate of patients with lymphovascular (L1) and perineural invasion (Pn1), compared to those with L0 and Pn0 status (LO/1 109.10; $95 \% \mathrm{Cl}, 94.73-123.46$ vs 47.76 ; $95 \% \mathrm{Cl}, 29.50-$ 66.02; $\mathrm{p}<0.001 ; \mathrm{PnO} / 1$ - 105.84; $95 \% \mathrm{Cl}, 90.58-121.11$ vs $52.49 ; 95 \% \mathrm{Cl}, 34.04-70.93 ; p<0.001)$.

\section{DISCUSSION}

P-NETs are rare tumours with heterogeneous biological behaviour, that are usually diagnosed with synchronous liver metastases in $25-93 \%$ of cases (1). Nowadays surgical resection is still the only curative option of treatment. The type of surgery is based mainly on tumour function, location, grading and staging. The standard anatomical resections pancreaticoduodenal resection and distal pancreatectomy with LND for NF-pNETs and pancreatic enucleation for small benign insulinomas, respectively,
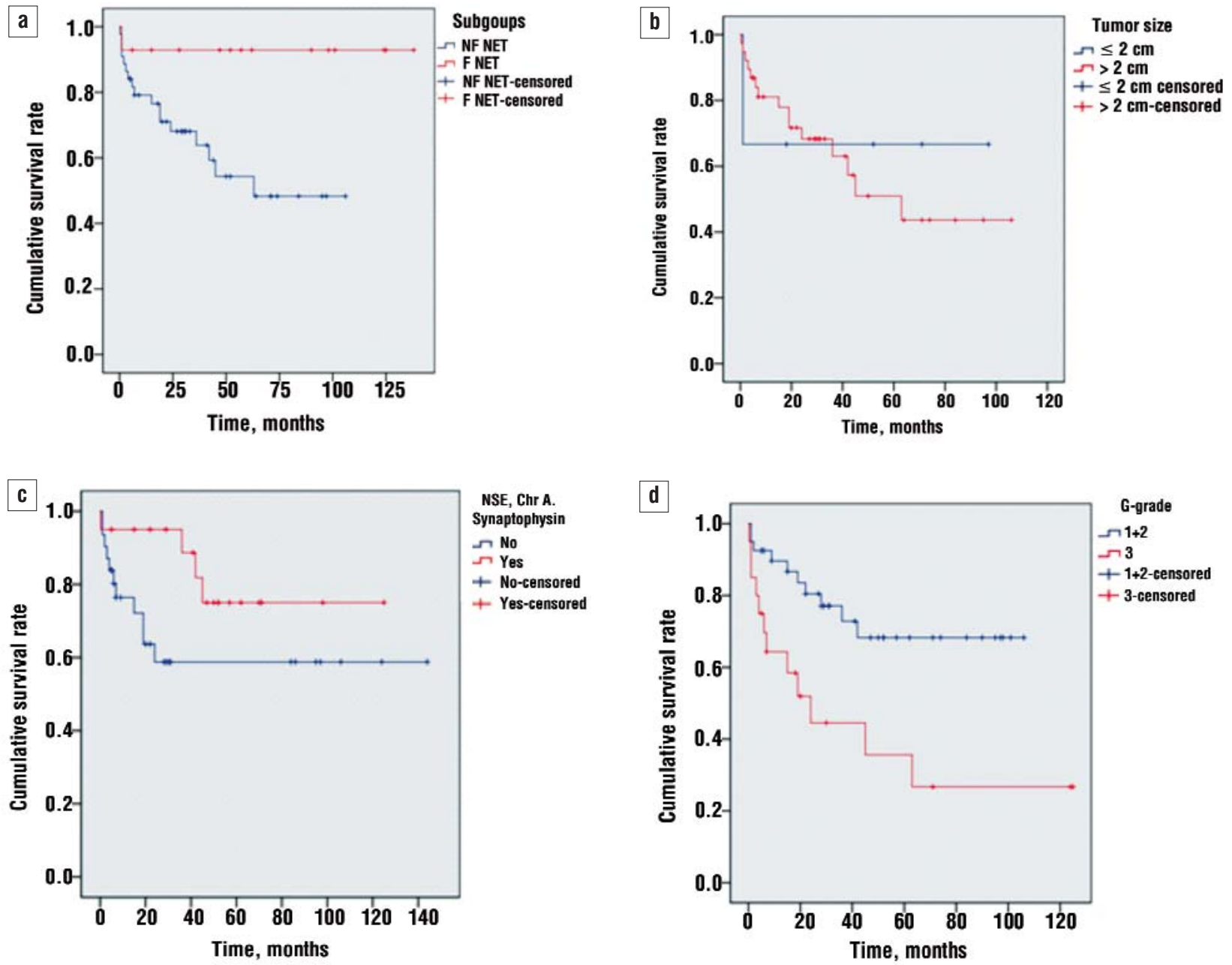

Figure 2 - Kaplan-Meier survival curves of patients with a) F-pNETs versus NF-pNETs, Log-rank test p-value 0.036 ; $b$ ) pNETs $\leq 2 \mathrm{~cm}$ vs pNETs $>2 \mathrm{~cm}$, log-rank test p-value 0.753 ; c) NSE, ChrA, synaptophysin "triple positive" pNETs vs "triple negative" pNETs, log-rank test p-value 0.083 ; d) pNETs G1+G2 vs pNETs G3; log-rank test p-value 0.004 
were the most frequent surgical procedures performed in our series. The overall morbidity rate in our study was comparable to rates of $40-50 \%$ in previously published studies (7). The 30 -day mortality was still high (5.17\%).

There was no statistically significant difference in the postoperative morbidity rate between enucleation, which we mainly use in insulinoma cases, and other types of surgical procedures in regards to the rate of POPF ( $p=0.132)$. The latter accounts for $32.7 \%$ and is the leading cause of mortality (66.67\%). There was no statistically significant difference in the postoperative morbidity rate between enucleation and pancreatoduodenal resection when the tumour was located in the head of the pancreas. However, when the tumour was located in the body/tail region, both early postoperative bleeding ( $p=0.033)$ and surgical site infections $(p=0.02)$ occurred more frequently in the enucleation group. Standard LND was not performed in our study in patients with insulinomas, considering the latter's mostly benign. Gastrinomas, on the other hand, are an indication for standard resection with LND (5). When enucleation is planned for a small pNET, a secretinstimulated MRI is performed as per protocol in order to measure tumour-to-main pancreatic duct distance. This allows us to choose the optimal type of intervention: if the distance is larger than 2-3 $\mathrm{mmm}$, enucleation is considered. In patients with a tumour situated in close proximity to the pancreatic duct, a standard resection will be performed in order to prevent complications such as POPF (8) or bleeding. Intraoperative US should also be available in the operating theatre whenever a patient with insulinoma is operated on. The individual estimation may require standard operative procedure. While some small $(\leq 2 \mathrm{~cm})$ pNETs can benefit from enucleation (8), we prefer a strict follow-up an "wait and watch" approach in cases of incidentaloma. Central pancreatectomy, although preferred in some centers (9), is not a choice in our Department due to relatively high morbidity rates. We prefer to perform either enucleation or distal pancreatectomy instead. Unfortunately, performing the aforementioned procedures laparoscopically does not lead to a decrease in the postoperative complication rate (10). Comparing open to laparoscopic DSP, no significant difference was found in the morbidity rate (notably POPF B+C$p=0.967$ and postoperative bleeding $-p=1.000$ ). There were insufficient laparoscopic enucleations to compare to the group of patients operated via the classical approach. Nevertheless, laparoscopic surgery for small pNETs appears to be feasible and safe when performed in specialized centres (11).

Compared to pancreatic adenocarcinoma, pNETs are less aggressive and patients with distant metastases may be treated with surgery whenever it is oncologically appropriate. Extended surgery is admissible in patients with low proliferation index of the primary tumour and with no extra-abdominal disease (12). We do practice an aggressive approach such as multi-visceral and vascular resections for locally advanced G1-2 pNETs and combined liver procedures for liver metastases on a strictly individually selected bias. It showed no statistical difference in morbidity and mortality rate, but the survival rate was proved to be poorer in vascular and combined liver resections. We ascribe these results as affected by the retrospective design and relatively small number of patients in our study as well as to other prognostic factors such as lymph-node ratio, undiscovered extra-liver metastases, etc. The fact that the introduction of $\mathrm{Ga}^{6}{ }^{68} \mathrm{PET} / \mathrm{CT}$ led to an unanticipated increase in the detection of extra-hepatic NET metastases (13) may lead to a change in therapeutic regimens and to a reconsideration of the indications for metastatic liver surgery.

According to a recently published meta-analysis, (14) R1-margin, advanced G- and TMN-stage, vascular invasion as well as organ metastases are associated with a poor prognosis of pNETs. Nevertheless, factors such as age, gender, surgical type and size of tumour could not be considered as prognostic factors. RO status was achieved in all patients who underwent radical procedures in our cohort thereby precluding the need to analyse R0/R1 status in the statistical analysis. Upon applying Cox-regression for prognostic factors' evaluation and a step-procedure, the only factors that were found as statistically significant predictors of poor outcome were non-functional status, distant metastases, vascular resection and lymphovascular invasion. Although some factors showed better overall survival, they were not statistically significant after multivariate analysis. While the relatively small number of patients in our cohort affects the results, the patients with the aforementioned negative prognostic factors should be strictly followed up and may require more aggressive therapeutic regimens.

\section{CONCLUSION}

The most effective treatment of the pancreatic neuroendocrine tumors is by means of the multidisciplinary approach in specialized centres. Radical surgery must be considered in all cases, if it is oncologically consistent and technically achievable. Anatomical resections (standard pancreaticoduodenal resection and distal pancreatectomy with standard 
lymph-node dissection) for NF-pNETS, as well as parenchyma-sparing operations (in particular tumour enucleation) for small benign insulinomas, are main surgical options in the treatment of pNETs.

\section{Conflict of interest}

\section{All author declare that they have no conflict of interest.}

\section{REFERENCES}

1. Cho ME, Kim JM, Sohn JH, Kim MJ, Kim MK, Woo Ho, et al. Current Trends of the Incidence and Pathological Diagnosis of Gastroenteropancreatic Neuroendocrine Tumors (GEP-NETs) in Korea 2000-2009: Multicenter Study. Cancer Res Treat. 2012; 44(3):157-165.

2. Metz DC, Jensen RT. Gastrointestinal neuroendocrine tumors: pancreatic endocrine tumors. Gastroenterology. 2008;135:1469- 92.

3. Bosman FT, Carneiro F, Hruban R, Theise N: WHO Classification of Tumours of the Digestive System, ed 4. International Agency for Research on Cancer (IARC), Lyon, 2010.

4. Edge SB, Byrd DR, Compton CC, Fritz AG, Greene FL, Trotti A (eds). AJCC Cancer Staging Manual. 7th ed. New York: Springer; 2010.

5. Falconi M, Bartsch DK, Eriksson B, Klöppel G, Lopes JM, O'Connor $\mathrm{JM}$, et al. ENETS Consensus guidelines for the management of patients with digestive neuroendocrine neoplasms of the digestive system: well differentiated pancreatic nonfunctioning tumors. Neuroendocrinology. 2012;95:120-34

6. Bassi C, Marchegiani G, Dervenis C, Sarr M, Abu Hilal M, Adham M, et al. The 2016 update of the International Study Group (ISGPS) definition and grading of postoperative pancreatic fistula: 11 Years After. Surgery. 2017;161(3):584-591.

7. Mayo SC, Gilson MM, Herman JM, Cameron JL, Nathan H, Edil BH, et al. Management of patients with pancreatic adenocarcinoma: national trends in patient selection, operative management, and use of adjuvant therapy. J Am Coll Surg. 2012;214(1):33-45.

8. Chua TC, Yang TX, Gill AJ, Samra JS. Systematic Review and Meta-Analysis of Enucleation Versus Standardized Resection for Small Pancreatic Lesions. Ann Surg Oncol. 2016; 23(2):592-9.

9. Bassi C, Middle segment pancreatectomy: a useful tool in the management of pancreatic neoplasms, J Gastrointest Surg, 2007; 11(4):421-424.

10. Limongelli P, Belli A, Russo G, Cioffi L, D’Agostino A, Fantini C, et al. Laparoscopic and open surgical treatment of left-sided pancreatic lesions: clinical outcomes and cost-effectiveness analysis. Surg Endosc. 2012;26(7): 1830-1836.

11. Mehrabi A, Hafezi M, Arvin J, Esmaeilzadeh M, Garoussi C, Emami $G$, et al. A systematic review and meta-analysis of laparoscopic versus open distal pancreatectomy for benign and malignant lesions of the pancreas: it's time to randomize. Surgery 2015;157:45-55

12. Fendrich V, Langer P, Celik I, Bartsch DK, Zielke A, Ramaswamy A, Rothmund M. An aggressive surgical approach leads to long-term survival in patients with pancreatic endocrine tumors. Ann Surg. 2006;244:845-51.

13. Albanus DR, Apitzsch J, Erdem Z, Erdem 0, Verburg FA, Behrendt FF, et al., Clinical value of Ga-DOTATATE-PET/CT compared to stand-alone contrast enhanced CT for the detection of extra-hepatic metastases in patients with neuroendocrine tumours (NET). Eur $\mathrm{J}$ Radiol. 2015 0ct;84(10):1866-72.

14. Gao Y, Gao H, Wang G, Yin L, Xu W, Peng Y, et al. A meta-analysis of Prognostic factor of Pancreatic neuroendocrine neoplasms. Sci Rep. 2018; 8: 7271. 